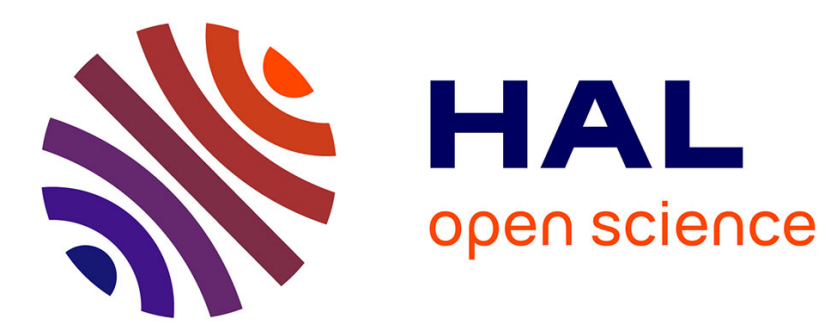

\title{
Theoretical description of metal/oxide interfacial properties: The case of $\mathrm{MgO} / \mathrm{Ag}(001)$
}

Stefano Prada, Livia Giordano, Gianfranco Pacchioni, Jacek Goniakowski

\section{To cite this version:}

Stefano Prada, Livia Giordano, Gianfranco Pacchioni, Jacek Goniakowski. Theoretical description of metal/oxide interfacial properties: The case of $\mathrm{MgO} / \mathrm{Ag}(001)$. Applied Surface Science, 2016, 390, pp.578-582. 10.1016/j.apsusc.2016.08.087 . hal-01362218

\section{HAL Id: hal-01362218 \\ https://hal.sorbonne-universite.fr/hal-01362218}

Submitted on 8 Sep 2016

HAL is a multi-disciplinary open access archive for the deposit and dissemination of scientific research documents, whether they are published or not. The documents may come from teaching and research institutions in France or abroad, or from public or private research centers.
L'archive ouverte pluridisciplinaire HAL, est destinée au dépôt et à la diffusion de documents scientifiques de niveau recherche, publiés ou non, émanant des établissements d'enseignement et de recherche français ou étrangers, des laboratoires publics ou privés. 


\title{
Theoretical description of metal/oxide interfacial
}

\section{properties: the case of $\mathrm{MgO} / \mathrm{Ag}(001)$}

\author{
Stefano Prada, Livia Giordano, ${ }^{*}$ Gianfranco Pacchioni \\ Dipartimento di Scienza dei Materiali, Università di Milano-Bicocca, via R. Cozzi, \\ 53, 20125 Milano, Italy \\ Jacek Goniakowski \\ CNRS-Sorbonne Universités UPMC Univ. Paris 06, UMR 7588, INSP, F-75005 \\ Paris, France
}

Version August 3, 2016

\begin{abstract}
:
We compare the performances of different DFT functionals applied to ultra-thin MgO(100) films supported on the Ag(100) surface, a prototypical system of a weakly interacting oxide/metal interface, extensively studied in the past. Beyond semi-local DFT-GGA approximation, we also use the hybrid DFT-HSE approach to improve the description of the oxide electronic structure. Moreover, to better account for the interfacial adhesion, we include the van de Waals interactions by means of either the semi-empirical force fields by Grimme (DFT-D2 and DFT-D2*) or the selfconsistent density functional optB88-vdW. We compare and discuss the results on the
\end{abstract}


structural, electronic, and adhesion characteristics of the interface as obtained for pristine and oxygen-deficient Ag-supported MgO films in the 1-4 ML thickness range.

Keywords: metal/oxide interface, oxide films, interfacial charge transfer, van de Waals interactions, hybrid functionals

* Corresponding author: e-mail: livia.giordano@mater.unimib.it

\section{Introduction}

The interfaces between oxides and metals are relevant in many fields, ranging from microelectronics and oxide-based magnetic devices, to heterogeneous catalysis and photocatalysis. ${ }^{1,2,3,4}$ In all applications, the nature of the interaction at metal/oxide interface is crucial for the properties of the combined system. For instance, the strength of adhesion between the two materials is linked to the interfacial distances and charge transfers, as strong chemical bonds at the interface produce large changes in the electronic distribution and shorter interface distances. The adhesion strength also determines the growth mode, the shape and the morphology in the case of supported nanoparticles or ultra-thin films. The characteristics of the interface also impact various electronic properties of the above-mentioned deposits. As an example, they drive the work function of metal-supported oxide ultra-thin films, which is particularly sensitive to the interfacial distance. ${ }^{5,6,7,8}$ On the other hand, the charge transfer at the interface induces a polarization of the supported oxide film, due to an electrostatic coupling between the film and the interfacial dipole moments. ${ }^{8}$ These combined mechanisms determine the surface properties of metal-supported oxide 
films, 7, 9,10, 11 making their chemical reactivity different from that of bulk oxide surfaces. ${ }^{12,13,14,15,16}$ A striking example is the spontaneous charging of adsorbates, enabled by the oxide-induced work function change, the structural flexibility of oxide films, and the electron reservoir provided by the underlying metal. ${ }^{17,18,19,20}$ Metal/oxide interface properties also determine the position of the Fermi level within the oxide band gap, eventually modifying the charge state of defects, such as oxygen vacancies (F centers), and thus altering the optical and chemical (catalytic) properties of metal-supported oxide films. As an example, while on the surfaces of bulk MgO neutral oxygen defects $\left(\mathrm{F}^{0}\right)$ are by far the most stable, charged vacancies $\left(\mathrm{F}^{+}\right)$may be favored in supported oxide films. This stabilization is due to a reduced substrate work function and to strong charge image effects in the thinnest metal-supported $\mathrm{MgO}$ films 21,22

The above examples elucidate the importance of an accurate description of metal/oxide interfaces for a valid assessment of the delicate balance between electronic and structural effects. ${ }^{23,24}$ Even though standard DFT has been routinely used in studies on interface characteristics, ${ }^{7,10,11}$ the question of the impact of its known drawbacks remains open. Indeed, while satisfactory for metals, (semi-)local exchange-correlation functionals fail to reproduce band gaps and positions of band edges in semiconducting and insulating oxides. ${ }^{25}$ Conversely, hybrid approaches, including a fraction of exact Hartree-Fock (HF) exchange, improve the description of oxides but are much less suited for metals, ${ }^{26}$ despite promising improvements brought by the range-separated hybrid functionals. ${ }^{27}$

Recently we have shown that both $\mathrm{DFT}+\mathrm{U}$ and hybrid functionals describe satisfactory the structural and electronic properties of $\mathrm{Pt}(111)$-supported $\mathrm{FeO}_{\mathrm{x}}(111)$ films, as well as the effect of the spontaneous charging of Au adatoms. ${ }^{28}$ Our present 
goal is to extend this analysis to the qualitatively different case of $\mathrm{MgO} / \mathrm{Ag}$ system, prototypical of a weakly interacting interface, extensively studied in the past. $^{29,30,31,18,32}$ Contrary to $\mathrm{FeO}_{\mathrm{x}}$, the electronic structure of MgO cannot be easily improved with the DFT+U method. Moreover, since the interaction between Ag and $\mathrm{MgO}$ is weak, the van de Waals interactions, absent in standard and hybrid DFT, may be essential for a correct description of this interface. To this goal we compare and discuss GGA (PBE) and hybrid (HSE06) results on interface structural, electronic, and adhesion characteristics for pristine and oxygen-deficient $\mathrm{MgO}(100)$ ultra-thin films (1-4 ML thick) on $\mathrm{Ag}(100)$ and confront them with calculations including van der Waals interactions, via both semi-empirical force field by Grimme, ${ }^{33}$ and selfconsistent vdW functionals. ${ }^{34,35}$

\section{Computational method}

Spin-polarized calculations have been performed in the framework of Density Functional Theory (DFT), using a plane-waves basis set as implemented in the VASP code. ${ }^{36,37}$ We employed semi-local GGA (PBE) and hybrid (PBE0 ${ }^{38}$ and HSE0 $6^{27,39,40}$ ) functionals. Van der Waals (wdW) interactions were accounted for by either the semi-empirical DFT-D2 method by Grimme (with PBE functional), ${ }^{33}$ or the density functional optB88-vdW. ${ }^{34,35}$ For the former we also considered the DFT-D2* modification, more suitable for ionic systems, with the less polarizable Ne used for $\mathrm{Mg}^{2+} \cdot 41$

The Ag(100) surface was represented by a slab composed of four atomic layers, with up to 4 monolayers (ML) of $\mathrm{MgO}(100)$ adsorbed on one side. A vacuum layer of about $12 \AA$ and dipole corrections were used in order to eliminate the spurious 
interactions between periodic replica. The oxide film is compressed (by 2\%) to match the Ag lattice parameter, in agreement with the pseudomorphic growth observed up to $5-7 \mathrm{ML} \mathrm{MgO} .^{29}$ We used a $(1 \times 1)$ surface unit cell in the calculations of pristine MgO films (MgO(nML)/Ag), while for films containing oxygen vacancies in the oxide surface layer we employed a $(2 \times 2)$ supercell, which represents a compromise between the computational effort and the strength of the lateral interactions between the vacancies. The positions of all atoms in the MgO film and in the two outmost metal layers were fully relaxed, until forces became smaller than $0.01 \mathrm{eV} / \AA$. The remaining two metal layers were frozen at their optimized bulk positions. The reciprocal space of the $(1 \times 1)$ surface cell was sampled with a $(11 \times 11)$ MonkhorstPack mesh, while a $(6 \times 6)$ mesh was used for the $(2 \times 2)$ cell. Atomic charges were estimated within the Bader decomposition scheme. ${ }^{42}$

\section{Results and discussion}

\subsection{Isolated $\mathrm{MgO}$ and $\mathrm{Ag}$}

Table 1 summarizes the calculated properties of isolated $\mathrm{MgO}$ and Ag to illustrate the strengths and shortcomings of the different methods in describing the two materials. Both structural (lattice parameter) and electronic (bulk band gap, position of surface valence ( $\left.\mathrm{IP}_{\text {surf }}\right)$ and conduction ( $\left.\mathrm{AE}_{\text {surf }}\right)$ band edges) characteristics of $\mathrm{MgO}$ are clearly better described with hybrid functionals, although PBE0 is required to fully approach the experimental band gap of $7.8 \mathrm{eV} .{ }^{43}$ DFT-D2 functional predicts lattice parameter close to the experimental value, while optB88 calculation gives an overestimated, PBE-like, value. Both van der Waals functionals do not bring an improvement of the band gap. 
While all considered methods overestimate the lattice parameter of Ag by a similar small amount of $1-2 \%$, the work function of the $\operatorname{Ag}(100)$ surface strongly depends on the method. The experimental value is best reproduced at the PBE and DFT-D2 level, while it is underestimated at hybrid level, and overestimated by the optB88 functional.

Table 1. Properties of MgO and Ag as a function of the method: lattice parameter (a), MgO bulk band gap $\left(\mathrm{E}_{\mathrm{g}}\right)$; MgO surface ionization potential (IP surf) and electron affinity $\left(\mathrm{AE}_{\text {surf }}\right)$; $\operatorname{Ag}(100)$ surface work function $(\phi)$.

\begin{tabular}{lcccccc}
\hline \multicolumn{9}{c}{ MgO } & \multicolumn{3}{c}{ Ag } \\
& $\mathrm{a}$ & $\mathrm{E}_{\mathrm{g}}$ & $\mathrm{IP}_{\text {surf }}$ & $\mathrm{AE}_{\text {surf }}$ & $\mathrm{a}$ & $\phi$ \\
& $(\AA)$ & $(\mathrm{eV})$ & $(\mathrm{eV})$ & $(\mathrm{eV})$ & $(\AA)$ & $(\mathrm{eV})$ \\
\hline PBE & 4.25 & 4.47 & -5.28 & -2.05 & 4.16 & 4.17 \\
PBE0 & 4.21 & 7.23 & - & - & 4.15 & 4.05 \\
HSE06 & 4.21 & 6.51 & -6.19 & -1.57 & 4.15 & 3.98 \\
DFT-D2 & 4.20 & 4.84 & -5.31 & -1.98 & 4.15 & 4.19 \\
optB88 & 4.24 & 5.10 & -5.50 & -2.30 & 4.14 & 4.53 \\
Exp. & 4.21 & 7.8 & $6.7^{44}$ & - & 4.09 & 4.2 \\
\hline
\end{tabular}

\subsection{Pristine $\mathrm{MgO}(3 M L) / \mathrm{Ag}$}

The structural and electronic characteristics of the $\mathrm{MgO}(3 \mathrm{ML}) / \mathrm{Ag}$ film are summarized in Table 2. As expected, the interface adhesion energy ( $\left.E_{a d h}\right)$ is very sensitive to the level of theory. In absence of the vdW contribution, both PBE and HSE06 calculations predict a weak $E_{\text {adh }}$ of $0.3-0.4 \mathrm{~J} / \mathrm{m}^{2}$. The adhesion energy obtained from DFT-D2 is nearly three times larger, while both DFT-D2* and optB88 predict intermediate values of $0.7-0.8 \mathrm{~J} / \mathrm{m}^{2}$. While to our knowledge there is no experimental 
estimate of the $\mathrm{MgO}$ adhesion energy on $\mathrm{Ag}(100)$, the interaction strength of $\mathrm{Ag}$ nano-particles with the $\mathrm{MgO}(100)$ surface has been studied in the past. From the analysis of cluster shapes observed by HRTEM, the adhesion energy was estimated to be $0.49-0.89 \mathrm{~J} / \mathrm{m}^{2}$, ${ }^{45}$ while a value of $0.75-0.85 \mathrm{~J} / \mathrm{m}^{2}$ was obtained by SDRS and GISAXS analyses for cluster sizes lower than $10 \mathrm{~nm} .{ }^{46,47}$ The discussion in Ref. 45 concluded on the value of $0.58 \pm 0.1 \mathrm{~J} / \mathrm{m}^{2}$ as a good reference for the ideal $\mathrm{Ag}(100) / \mathrm{MgO}(100)$ interface. Although none of the computed values closely reproduces this estimate, the DFT-D2* and optB88 results are in much better agreement. Conversely, DFT-D2 severely overestimates the interfacial adhesion and PBE and HSE06 approximations underestimate it. Similar conclusions have been reported for the related $\mathrm{Ag} / \mathrm{MgO}(100)$ system. $^{24}$

Consistently with the calculated adhesion energies, PBE and HSE06 predict similarly large interface distances, while with semi-empirical DFT-D2 and DFT-D2* methods the MgO layer is much closer to the Ag surface. Interestingly, the optB88 functional strengthens the interfacial adhesion with only a little effect on the interfacial distance. We note that the DFT-D2 and DFT-D2* values are the closest to the experimental estimate of 2.4-2.5 $\AA,{ }^{22,31,29}$ although other effects, such as the finite size of the islands $^{23}$ may also influence the experimental interfacial distance. Other structural properties, such as the oxide film rumpling, are less dependent on the method used, with a somewhat larger rumpling obtained with the Grimme's approach, consistent with the shorter interface distance. ${ }^{8}$

Table 2. Properties of $\mathrm{MgO}(3 \mathrm{ML}) / \mathrm{Ag}(100)$ films computed at different level of theory: adhesion energy per surface area $\left(\mathrm{E}_{\mathrm{adh}} / \mathrm{S}\right)$; interfacial distance $\left(\mathrm{d}_{\text {inter }}\right)$; $\mathrm{MgO}$ rumpling $\left(\mathrm{r}_{\mathrm{MgO}}\right)$, 
defined as the average intra-layer separation $\mathrm{z}_{\mathrm{O}}-\mathrm{Z}_{\mathrm{Mg}}$ (a positive value indicates oxygen outwards); charge transfer per interfacial $\mathrm{MgO}$ unit $\left(\mathrm{CT} / \mathrm{MgO}_{\mathrm{int}}\right.$, a positive value indicates electron transfer from $\mathrm{MgO}$ to $\mathrm{Ag})$; work function change with respect to the $\mathrm{Ag}(100)$ $\operatorname{surface}(\Delta \phi)$.

\begin{tabular}{lccccc}
\hline & $\mathrm{E}_{\text {adh }} / \mathrm{S}$ & $\mathrm{d}_{\text {inter }}$ & $\mathrm{r}_{\mathrm{MgO}}$ & $\mathrm{CT}$ & $\Delta \phi$ \\
& $\left(\mathrm{J} / \mathrm{m}^{2}\right)$ & $(\AA)$ & $(\AA)$ & $\left(\mathrm{e} / \mathrm{MgO}_{\text {int }}\right)$ & $(\mathrm{eV})$ \\
\hline PBE & 0.36 & 2.70 & 0.018 & 0.041 & -1.18 \\
HSE06 & 0.34 & 2.71 & 0.014 & 0.026 & -1.17 \\
DFT-D2 & 1.25 & 2.49 & 0.027 & 0.062 & -1.34 \\
DFT-D2* & 0.77 & 2.57 & 0.024 & 0.050 & -1.28 \\
optB88 & 0.76 & 2.65 & 0.017 & 0.046 & -1.41 \\
\hline
\end{tabular}

The calculated work function changes, $\Delta \phi$, Table 2 , confirm the reported reduction of Ag(100) work function induced by MgO films.55,6,22 While PBE and HSE06 predict a similar decrease of about $1.1 \mathrm{eV}$, the inclusion of $\mathrm{vdW}$ interactions enhances the effect to $1.3-1.4 \mathrm{eV}$. The amplitude of $\Delta \phi$ correlates with the interfacial distance, pointing to the compression of the electron density at the interface caused by the insulating film as the main cause of the observed effect. ${ }^{5,6}$ However, also the dipole moments due to the film rumpling and interfacial charge transfer tend to increase/decrease of the work function, respectively. Indeed, the largest $\Delta \phi$ obtained with optB88 despite a moderate interface distance, is due to a combination of a large interfacial charge transfer and a small film rumpling within this approximation. 
The density of states (DOS) of the $\mathrm{MgO}(3 \mathrm{~L}) / \mathrm{Ag}(100)$ film, Figure 1, highlights the expected sensitivity of the electronic structure of the interface on the exchangecorrelation functional. As expected, the HSE06 results differ substantially from the others: the $\mathrm{MgO}$ valence band edge and the Ag $3 d$ band are much lower in energy, while the Fermi level is visibly closer to the vacuum level. At the energy scale of Fig. 1, the PBE and both vdW-corrected DOS are very similar, although a small downshift of the top of the MgO O-2p band can be observed in optB88.

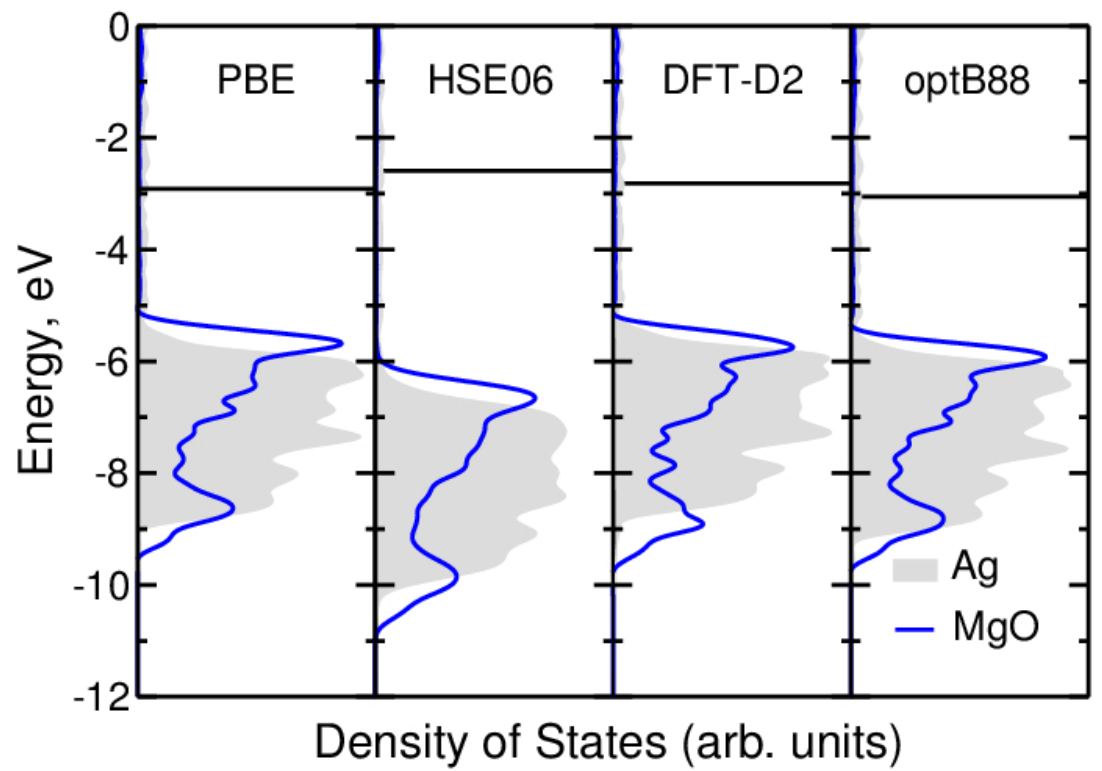

Figure 1 - MgO- and Ag-projected density of states of $\mathrm{MgO}(3 \mathrm{ML}) / \mathrm{Ag}(100)$ computed with PBE, HSE06, DFT-D2, and optB88 functionals. The zero energy is set to the vacuum level; the Fermi levels are indicated with horizontal lines.

\subsection{Pristine $\mathrm{MgO}(\mathrm{nML}) / \mathrm{Ag}$ : dependence on the film thickness}

Figure 2 summarizes the behavior of the structural and electronic characteristics in MgO films of thickness $n=1-4$ ML. We find that all the considered methods predict that the MgO monolayer substantially differs from the thicker films: the interface distance is visibly shorter, the film rumpling is larger, and the reduction of the surface 
work function is less pronounced. Beyond $1 \mathrm{ML}$, in the thickness range of $n=2-4$ ML, the film characteristics vary only slightly and remain similar to those of the $\mathrm{MgO}(3 \mathrm{ML}) / \mathrm{Ag}(100)$ case discussed above.

However, we find that the difference between the monolayer and thicker films may strongly depend on the method. This method-specific behavior is best exemplified by the weak dependence of the interfacial distance on the film thickness in methods including the van der Waals interaction, and in particular in the DFT-D2 approach, which predicts essentially a thickness-independent $d_{\text {int }}$. Interestingly, the behavior of the interface distance is not directly coupled to that of the film rumpling, for which all methods predict consistently a substantial (larger than 50\%) increase of rumpling at the monolayer thickness, driven by the reduced coordination of the ions and the enhanced flexibility of the single MgO layer. The enhanced rumpling in the monolayer, with anions relaxing strongly outwards, creates a dipole moment which increases the work function, and thus counteracts the reduction of $\phi$ induced by the MgO film. Indeed, $\Delta \phi$ is systematically smaller in the limit of a monolayer thickness independently of the method. We note that the decrease of $\Delta \phi$ as a function of tfilm thickness is the largest in DFT-D2 and DFT-D2* approximations, coherent with the small reduction of the interface distance predicted by these approaches.

Changes of the interfacial charge transfer are small and at the limit of the precision of the Bader decomposition method. We note that the charge transfer is the largest in DFT-D2 and DFT-D2*, coherent with the shortest interfacial distances. The other approaches tend to predict an increase of charge transfer at the monolayer thickness, which is linked to the shortening of $d_{\text {int }}$. 

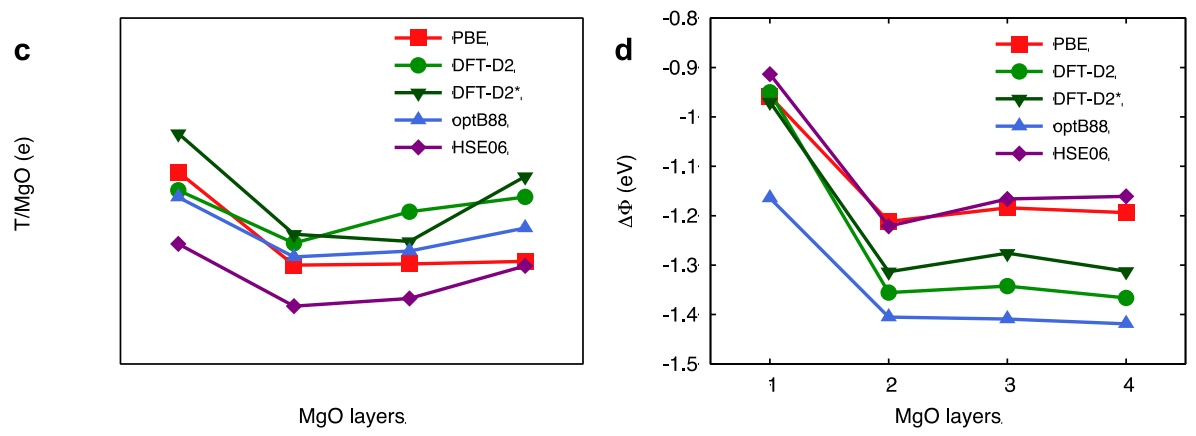

Figure 2. Properties of the $\mathrm{MgO}(\mathrm{nML}) / \mathrm{Ag}(100)$ interface as a function on the film thickness (n=1-4 ML), computed with the different methods: (a) interface distance; (b) charge transfer; (c) MgO rumpling; (d) work function change with respect to the $\mathrm{Ag}(100)$ surface.

\subsection{Surface vacancies at $\mathrm{MgO}(\mathrm{nML}) / \mathrm{Ag}$ : properties and dependence on film thickness}

Table 3 summarizes the results for the neutral oxygen vacancy on the surface of $\mathrm{MgO}(3 \mathrm{ML}) / \mathrm{Ag}$. At all levels of theory the small charge transfer towards the substrate and the relatively weak structural relaxation around the point defect indicate that the vacancy is neutral, with two electrons trapped in cavity in a singlet state, in agreement with the existing GGA results for this system. ${ }^{21}$ We notice that the somewhat larger charge transfer and structural relaxation of ions around the vacancy predicted by PBE and optB88 do not alter the singlet electronic state of trapped electrons. More interestingly, our results show that either an improved description of the oxide band structure (exact exchange in HSE) or a better atomic structure of the interface (shorter 
interface distances in DFT-vdW) do not shift the vacancy electronic state enough to promote an electron transfer towards the Ag substrate.

Similar electronic characteristics result in substantially similar vacancy formation energies $\left(E_{\text {form }}\right)$ predicted by all the considered approaches. We note that $E_{\text {form }}$ reported in Table 3 includes also the contribution due to a different quality of description of the isolated oxygen molecule. In particular, smaller $\mathrm{E}_{\text {form }}$ predicted by GGA compared to that by HSE is linked to the well known over-binding of the $\mathrm{O}_{2}$ molecule in the former.

Table 3. Properties of the oxygen vacancy at the surface of $\mathrm{MgO}(3 \mathrm{ML}) / \mathrm{Ag}(100)$ film computed at different level of theory: formation energy with respect to gas phase $1 / 2 \quad \mathrm{O}_{2}$ $\left(E_{\text {form }}\right)$; relaxation around the defect, computed as the in-plane displacement of the cations around the defect with respect to the pristine film (rel); charge transfer per defect (CT), work function change with respect to the $\mathrm{MgO}(3 \mathrm{ML}) / \mathrm{Ag}(100)$ film ( $\left.\Delta \phi_{\text {vac }}\right)$.

\begin{tabular}{lcccc}
\hline & $\begin{array}{c}\mathrm{E}_{\text {form }} \\
(\mathrm{eV})\end{array}$ & $\begin{array}{c}\mathrm{CT} \\
(\%)\end{array}$ & $\begin{array}{c}\Delta \phi_{\text {vac }} \\
(e / \text { defect })\end{array}$ & $(\mathrm{eV})$ \\
\hline PBE & 6.11 & 1.9 & 0.14 & +0.32 \\
HSE06 & 6.50 & 1.4 & 0.07 & +0.53 \\
DFT-D2 & 6.35 & 1.5 & 0.08 & +0.33 \\
optB88 & 6.75 & 1.7 & 0.17 & +0.41 \\
\hline
\end{tabular}

It is worth noticing that, while all methods predict a vacancy-induced increase of $\mathrm{MgO}(3 \mathrm{ML}) / \mathrm{Ag}$ work function (positive $\Delta \phi_{\text {vac }}$ in Table 3), this effect is considerably larger in HSE than in the other approximations. Since the increase of $\phi$ is mainly assigned to the spill out of the vacancy electrons density into the vacuum, its strengthening in the case of HSE is on the other hand connected to an enhanced 
surface electrostatic field within the hybrid method driven by a larger ionization potential (IP surf in Table 1).

The characteristics of the surface oxygen vacancy as a function of the film thickness are reported in Figure 3. All methods concur in indicating a progressive change of the defect nature with decreasing film thickness, as principally witnessed by the dramatic increase of charge transfer towards the Ag substrate. Indeed, at 1ML thickness one of the two electrons trapped in the vacancy is transferred to the metal, producing a positively charged defect $\left(\mathrm{F}^{+}\right)$in the MgO film. Interestingly, at difference with $\mathrm{F}^{+}$in bulk MgO, and despite the inclusion of the exact exchange in HSE, the hybridization between vacancy and metal states causes this defect to be non-magnetic, giving thus support to the existing GGA results. ${ }^{21,23,48}$ Beyond the concurrent description of electron delocalization, all considered approximations give also a fairly similar picture of the vacancy in the monolayer, with an increase of film relaxation, a drop of vacancy formation energy, and a reduction of the work function compared to the 3ML case.

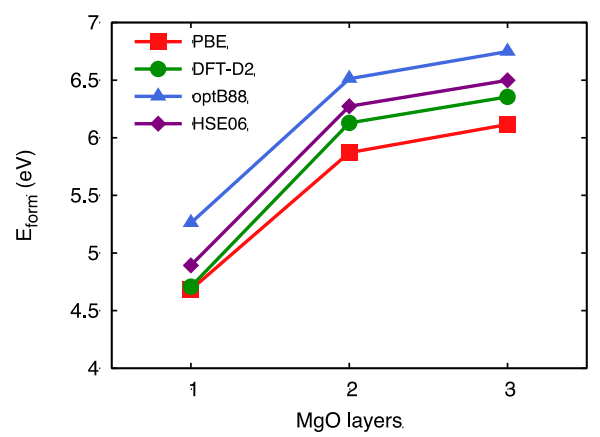


Figure 3. Properties of the surface oxygen vacancy on $\mathrm{MgO}(\mathrm{nML}) / \mathrm{Ag}(100)$ films as a function on the thickness ( $=1-3 \mathrm{ML}$ ), computed with the different methods: (a) formation energy $\left(E_{\text {form}}\right)$; (b) charge transfer per defect; (c) relaxation around the defect, computed as the lateral displacement of the cations around the defect with respect to the pristine film (rel); (d) work function change with respect to the case of vacancy in $\mathrm{MgO}(1 \mathrm{ML}) / \mathrm{Ag}(100)$ film $\left(\Delta \phi_{\mathrm{vac}}\right)$.

Taking into account the different nature of the oxygen vacancy in 3ML and $1 \mathrm{ML}$ films, the intermediate $\mathrm{MgO}(2 \mathrm{ML}) / \mathrm{Ag}$ case could have been expected to be particularly sensitive to the quality of description of the interface electronic and atomic structure. This, however, is clearly not the case, since all calculations, regardless the level of approximation, predict an ensemble of very similar characteristics. Similarly to the reported results for the $\mathrm{FeO}_{x} / \mathrm{Pt}$ system, ${ }^{28}$ also in the present case the changes in the band alignment at the interface induced by improvements of either electronic or atomic structures are not sufficient to qualitatively alter the nature of the interface and the quantitative changes of its characteristics are very limited.

\section{Conclusions}

We have used a variety of DFT functionals: GGA, HSE, DFT-D2 and optB88-vdW to study ultra-thin $\mathrm{MgO}(100)$ films weakly interacting with the $\operatorname{Ag}(100)$ substrate, and compared their performance on the structural, electronic, and adhesion characteristics for pristine and oxygen-deficient MgO films in the thickness range of 1-4 ML.

The large differences in the description of the separate $\mathrm{MgO}$ and Ag systems with the various approaches affect the characteristics of the $\mathrm{MgO} / \mathrm{Ag}$ interface to a different extent. The most method-sensitive properties are the adhesion energy and the 
interfacial distance, for which the experimental estimates are satisfactorily approached by functionals including the van der Waals interactions (DFT-D2* and optB88), while PBE and HSE06 tend to underestimate the strength of interfacial interactions. We also notice that the standard DFT-D2 seems to severely overestimate the adhesion energy.

The other properties, including the trends as a function of the film thickness are much less affected by the choice of the computational method. This is particularly striking in the case of surface oxygen vacancies, for which all methods consistently predict a progressive evolution of the vacancy nature from "neutral" in thicker films to “charged” in the limit of a single MgO monolayer.

Similarly to the $\mathrm{FeO}_{\mathrm{x}} / \mathrm{Pt}$ system, ${ }^{28}$ the changes in the interface band alignment induced by the more accurate description of the electronic or atomic structure with hybrid or vdW-including functionals do not alter the nature of the interface and produce limited changes of its fine characteristics.

\section{Acknowledgments}

We are grateful to Claudine Noguera for insightful discussions. We acknowledge support from the COST Action CM1104 Reducible oxide chemistry, structure and functions and in particular the funding of a Short Term Scientific Mission (S. P.). The work of S. P., L. G. and G. P. is also supported by the Italian MIUR through the FIRB Project RBAP115AYN.

\footnotetext{
${ }^{1}$ E. P. Gusev, Defects in $\mathrm{SiO}_{2}$ and related dielectrics: science and technology, G. Pacchioni, L. Skuja and D. L. Griscom (Eds.), Kluwer, Dordrecht, 2000, p. 557.
} 
${ }^{2}$ S. S. P. Parkin, C. Kaiser, A. Panchula, P. M. Rice, B. Hughes, M. Samant, S. H. Yang, Nature Mat. 3 (2004) 862.

${ }^{3}$ C. G. Grandqvist, Solar En. Mater. \& Solar Cell 91 (2007) 1529.

${ }^{4}$ L. M. Molina, B. Hammer, Phys. Rev. Lett. 90 (2003) 206102.

${ }^{5}$ L. Giordano, F. Cinquini, G. Pacchioni, Phys. Rev. B 73 (2006) 045414.

${ }^{6}$ S. Prada, U. Martinez, G. Pacchioni, Phys. Rev. B 78 (2008) 235423.

${ }^{7}$ L. Giordano, G. Pacchioni, Acc. Chem. Res. 44 (2011) 1244.

${ }^{8}$ J. Goniakowski, C. Noguera, Phys. Rev. B 79 (2009)155433.

${ }^{9}$ N. Nilius, Surf. Sci. Reports 64 (2009) 595.

10 S. Surnev, A. Fortunelli, F. P. Netzer, Chem. Rev. 113 (2013) 4314.

${ }^{11}$ C. Noguera, J. Goniakowski, Chem. Rev. 113 (2013) 4073-4105.

12 A. Hellman, S. Klacar, H. Grönbeck, J. Am. Chem. Soc. 131 (2009) 16636-16637.

${ }^{13}$ Y.-N. Sun, L. Giordano, J. Goniakowski, M. Lewandowski, Z.-H. Qin, C. Noguera, S. Shaikhutdinov, G. Pacchioni, H.-J. Freund, Angew. Chem. Int. Ed. 49 (2010) 44184421.

${ }^{14}$ H.-Y. T. Chen, L. Giordano, G. Pacchioni, J. Phys. Chem. C 117 (2013) 10623.

${ }^{15}$ L. Giordano, G. Pacchioni, C. Noguera, J. Goniakowski, ChemCatChem 6 (2014) 185.

${ }^{16}$ L. Giordano, A.M. Ferrari, J. Phys. Chem. C 116 (2012) 20349.

${ }^{17}$ G. Pacchioni, L. Giordano, M. Baistrocchi, Phys. Rev. Lett. 94 (2005) 226104.

${ }^{18}$ M. Sterrer, T. Risse, U. M. Pozzoni, L. Giordano, M. Heyde, H. P. Rust, G. Pacchioni, H.-J. Freund, Phys. Rev. Lett. 98 (2007) 096107.

${ }^{19}$ H. Grönbeck, J. Phys. Chem. B 110 (2007) 11977-11981.

${ }^{20}$ P. Frondelius, A. Hellman, K. Honkala, H. Hakkinen, H. Gronbeck, Phys. Rev. B 78 (2008) 085426.

${ }^{21}$ L. Giordano, U. Martinez, G. Pacchioni, M. Watkins, A. L. Shluger, J. Phys. Chem. C 112 (2008) 3857-3865.

${ }^{22}$ S. Ling, M. B. Watkins, A. L. Shluger, Phys. Chem. Chem. Phys. 15 (2013) 1961519624

${ }^{23}$ S. Ling, M. B. Watkins, A. L. Shluger, J. Phys. Chem. C 117 (2013) 5075-5083.

${ }^{24}$ B. He, D. Ma, W. Hao, W. Xiao, Z. Tian, Appl. Surf. Sci. 288 (2014) 115-121.

25 J. Muscat, A. Wander, N. M. Harrison, Chem. Phys. Lett. 342 (2001) 397-401.

${ }^{26}$ J. Paier, M.Marsman, G. Kresse, J. Chem. Phys. 127 (2007) 024103. 
${ }^{27}$ J. Heyd, G. E. Scuseria, M. Ernzerhof, J. Chem. Phys. 118 (2003) 8207-8215.

${ }^{28}$ S. Prada, L. Giordano, G. Pacchioni, C. Noguera, J. Goniakowski, J. Chem. Phys. 141 (2014) 144702.

${ }^{29}$ S. Valeri, S. Altieri, A. di Bona, P. Luches, C. Giovanardi, T. S. Moia, Surf. Sci. 507-510 (2002) 311.

${ }^{30}$ C. Giovanardi, A. di Bona, T. S. Moia, S. Valeri, C. Pisani, M. Sgroi, M. Busso, Surf. Sci. 505 (2002) L209-L214.

${ }^{31}$ P. Luches, S. D’Addato, S. Valeri, E. Groppo, C. Prestipino, C. Lambert, F. Boscherini, Phys. Rev. B 69 (2004) 045412.

32 J. Pal, M. Smerieri, E. Celasco, L. Savio, L. Vattuone, M. Rocca Phys. Rev. Lett., 112 (2014) 126102.

33 S. Grimme, J. Comput. Chem. 27 (2006) 1787-1799.

${ }^{34}$ M. Dion, H. Rydberg, E. Schroder, D. C. Langreth, B. I. Lundqvist, Phys. Rev. Lett. 92 (2004) 246401.

35 J. Klimeš, D. R. Bowler, A. Michaelides, J. Phys.-Condens. Mat. 22 (2010) 022201.

${ }^{36}$ G. Kresse, J. Hafner Phys Rev B 47 (1993) R558.

${ }^{37}$ G. Kresse, J. Furthmüller Phys Rev B 54 (1996) 11169.

${ }^{38}$ C.;Adamo, V. Barone, J. Chem. Phys. 110 (1999) 6158-6170.

${ }^{39}$ A. Stroppa, G. Kresse, New J. Phys. 10 (2008) 063020.

${ }^{40}$ M. Marsman, J. Paier, A. Stroppa, G. Kresse, J. Phys.: Condens. Matter 20 (2008) 064201

${ }^{41}$ S. Tosoni, J. Sauer, Physical Chemistry Chemical Physics 12 (2010) 14330.

${ }^{42}$ R. F. W. Bader, Chem. Rev. 91 (1991) 983; G. Henkelman, A. Arnaldsson, H. Jonsson, Comput. Mater. Sci. 36 (2006) 354; W. Tang, E. Sanville, and G. Henkelman, J. Phys.: Condens. Matter 21 (2009) 084204.

${ }^{43}$ D. M. Roessler, W. C. Walker Phys. Rev. 159 (1967) 733-738.

${ }^{44}$ P. V. Sushko, A. L. Shluger, C. R. A. Catlow, Surf. Sci. 450 (2000) 153-170.

${ }^{45}$ S. Stankic, R. Cortes-Huerto, N. Crivat, D. Demaille, J. Goniakowski, J. Jupille, Nanoscale 5 (2013) 2448.

${ }^{46}$ R. Lazzari, G. Renaud, C. Revenant, J. Jupille and Y. Borensztein, Phys. Rev. B: Condens. Matter Mater. Phys. 79 (2009) 125428.

${ }^{47}$ C. Revenant, G. Renaud, R. Lazzari and J. Jupille, Phys. Rev. B: Condens. Matter Mater. Phys. 79 (2009) 235424. 
${ }^{48}$ L. Giordano, J. Goniakowski, G. Pacchioni, Phys. Rev. B 67 (2003) 045410. 